Bull. Austral. Math. Soc.

$34 \mathrm{G} 10,39 \mathrm{~A} 12$

VoL. 53 (1996) [459-467]

\title{
REMARKS ON BOUNDED SOLUTIONS OF LINEAR SYSTEMS
}

Elena Topuzu and Paul Topuzu

In the case of continuous time systems with bounded operators (coefficients) the following result, of Perron type is well known: "The linear differential system $\dot{\boldsymbol{x}}=A x+f(t)$ has, for every function $f$ continuous and bounded on $\mathbb{R}$, a unique bounded solution on $\mathbb{R}$, if and only if the spectrum of the operator $A$ has no points on the imaginary axis".

In this paper we give a discrete version of this result. The case of continuous time systems with $A$ an unbounded, infinitesimal generator of a $C_{0}$ group, is considered in the last section.

\section{Preliminaries}

Let $X$ be a complex Banach space. We denote by $\mathcal{B}(X)$ the Banach algebra of all bounded linear operators from $X$ into itself. If $A \in \mathcal{B}(X)$ and $I$ is the unity of $\mathcal{B}(X)$ then

$$
\rho(A)=\left\{\lambda \in \mathbb{C}: \exists(\lambda I-A)^{-1}=R(\lambda, A) \in \mathcal{B}(X)\right\}
$$

and

$$
\sigma(A)=\{\lambda \in \mathbb{C}: \lambda \notin \rho(A)\}
$$

will denote respectively, the resolvent set and the spectrum of the operator $A$.

Let now $A: X \rightarrow X$ a bounded linear operator. For every continuous function $f: \mathbb{R} \rightarrow X$ we consider the differential equation

$$
(A, f) \quad \dot{x}=A x+f(t) .
$$

Connections between admissibility, asymptotical behaviours of solutions of the system $(A, f)$ and some spectral properties of the operator $A$, have been studied by many authors $[1,2,3,4]$.

It is known (see for example, $[1,2])$ that $(A, f)$ has a unique bounded solution on $\mathbb{R}$, for every $f$ continuous and bounded on $\mathbb{R}$, if and only if

$$
i \mathbb{R}=\{\lambda \in \mathbb{C}: \operatorname{Re} \lambda=0\} \subset \rho(A) .
$$

In this paper, using a discrete Green's function (constructed in Section 2), a similar result is given for linear discrete time systems.

Section 3 is concerned with the case when $A$ is unbounded. In this case we do not know if (1.1) is a sufficient condition to assure the validity of the previous result.

Received 27th July, 1995.

Copyright Clearance Centre, Inc. Serial-fee code: 0004-9729/96 \$A2.00+0.00. 


\section{BOUNDED SOLUTIONS OF LINEAR DISCRETE TIME SYSTEMS}

Let us consider $A \in \mathcal{B}(X)$ and

$$
f=\left(f_{n}\right)_{n \in Z}=\left(\ldots, f_{-2}, f_{-1}, f_{0}, f_{1}, f_{2}, \ldots\right)
$$

a $\mathbb{Z}$-indexed sequence of elements from $X$.

If $0 \in \rho(A)$ then it is easy to see that the linear discrete time system

$$
(A, f)_{d} \quad x_{n+1}=A x_{n}+f_{n}
$$

has solution $\left(x_{n}\right)_{n \in Z}$ given by

$$
x_{n}= \begin{cases}A^{n} x_{0}+\sum_{j=1}^{n-1} A^{n-j-1} f_{j}, & \text { for } n \geqslant 1 \\ A^{n} x_{0}+\sum_{j=n}^{-1} A^{n-j-1} f_{j}, & \text { for } n<-1\end{cases}
$$

uniquely determined by the value $x_{0} \in X$. In particular, if $f_{n}=0$ for every $n \in \mathbb{Z}$ then $\left(x_{n}=A^{n} x_{0}\right)_{n \in Z}$ is the unique solution for the discrete time system

$$
x_{n+1}=A x_{n}, \quad x_{0} \in X .
$$

Throughout this section $A$ will be a bounded linear operator with the property $0 \in \rho(A)$.

Also we denote

$$
\ell^{\infty}(\mathbb{Z}, X)=\left\{x=\left(x_{n}\right)_{n \in Z}:\|x\|=\sup _{n}\left\|x_{n}\right\|<\infty\right\}
$$

and

$$
\begin{aligned}
& D_{1}=\{\lambda \in \mathbb{C}:|\lambda|<1\} \\
& C_{1}=\{\lambda \in \mathbb{C}:|\lambda|=1\}
\end{aligned}
$$

Proposition 2.1. If $\sigma(A) \cap C_{1}=\emptyset$ and $\left(x_{n}=A^{n} x_{0}\right)_{n \in Z}$ is a solution of the system $(A)_{d}$ with $x_{0} \neq 0$ then $\left(x_{n}\right)_{n \in Z} \notin \ell^{\infty}(\mathbb{Z}, X)$.

Proof: Let us suppose that

$$
x=\left(x_{n}\right)_{n \in \mathbf{Z}}=\left(A^{n} x_{0}\right)_{n \in Z} \in \ell^{\infty}(\mathbb{Z}, X),
$$

that is

$$
\|x\|_{\infty}=\sup _{n \in Z}\left\|x_{n}\right\|<\infty
$$


(i) If $\sigma(A) \subset D_{1}$ then we find a positive number $a<1$ such that

$$
\sigma(A) \subset\{\lambda \in \mathbb{C}:|\lambda|<a<1\}
$$

and hence (see for example, [5]) we can find $N>0$ such that

$$
\left\|A^{n}\right\|<N a^{n}, \text { for every } n>0,
$$

from where, in particular, we obtain that:

$$
\sup _{n>0}\left\|A^{n} x\right\|<\infty, \quad \text { for every } x \in X .
$$

Since for $m<0, \quad x_{m}=A^{m} x_{0}$, we have

$$
\left\|x_{0}\right\|=\left\|A^{-m} x_{m}\right\| \leqslant\left\|A^{-m}\right\| \cdot\left\|x_{m}\right\| \leqslant N a^{-m} \cdot\|x\|_{\infty}, \quad \forall m<0
$$

from where we obtain that $x_{0}=0$.

(ii) If

$$
\sigma(A) \subset \operatorname{Ext}\left(D_{1}\right)=\{\lambda \in \mathbb{C}:|\lambda|>1\}
$$

then $\sigma\left(A^{-1}\right) \subset D_{1}$ and

$$
\sup _{n \in Z}\left\|A^{n} x_{0}\right\|=\sup _{n \in Z}\left\|A^{-n} x_{0}\right\|=\sup _{n \in Z}\left\|\left(A^{-1}\right)^{n} x_{0}\right\| .
$$

Hence, using case (i),

$$
\left(A^{n} x_{0}\right)_{n \in Z} \in \ell^{\infty}(\mathbb{Z}, X)
$$

if and only if $x_{0}=0$. Moreover it follows that

$$
\sup _{n>0}\left\|\left(A^{-1}\right)^{n} x\right\|=\sup _{n<0}\left\|A^{n} x\right\|<\infty, \quad \text { for every } x \in X .
$$

(iii) Finally, if

$$
\sigma(A)=\sigma_{1} \cup \sigma_{2}, \sigma_{1} \neq \emptyset, \sigma_{2} \neq \emptyset \text { and } \sigma_{1} \subset D_{1}, \sigma_{2} \subset \operatorname{Ext}\left(D_{1}\right)
$$

then, by the Dunford functional calculus, we can define the coresponding spectral projections

$$
P_{1}=\frac{1}{2 \pi i} \int_{\Gamma_{1}} R(\lambda, A) d \lambda, \quad P_{2}=\frac{1}{2 \pi i} \int_{\Gamma_{2}} R(\lambda, A) d \lambda
$$

where, for $j=1,2, \quad \Gamma_{j}$ is the boundary of a Cauchy domain $D_{j}$ which contains $\sigma_{j}$ and $\bar{D}_{1} \cap \bar{D}_{2}=0$. 
It is well known $[2,6]$ that $P_{1}+P_{2}=I, \quad P_{1} P_{2}=0$ and if $X_{j}=P_{j} X, j=1,2$ then both the subspaces $X_{1}$ and $X_{2}$ are invariant under the operator $A$. Moreover if $A_{j}$ is the restriction of $A$ to the subspace $X_{j}$ then $\sigma\left(A_{j}\right)=\sigma_{j}, j=1,2$.

Let now $\left(x_{n}\right)_{n \in Z}=\left(A^{n} x_{0}\right)_{n \in Z}$ be arbitrary solution of the system $(A)_{d}$. Then for every $n \in \mathbb{Z}$ we can write:

$$
x_{n}=A^{n}\left(P_{1}+P_{2}\right) x_{0}=A^{n} P_{1} x_{0}+A^{n} P_{2} x_{0}=A_{1}^{n} P_{1} x_{0}+A_{2}^{n} P_{2} x_{0}
$$

If $P_{1} x_{0}=0$ (or $P_{2} x_{0}=0$ ) then $x_{n}=A_{2}^{n} P_{2} x_{0}$ (respectively $x_{n}=A_{1}^{n} P_{1} x_{0}$ ) and by (ii) (respectively by (i)) the boundedness of $\left(x_{n}\right)_{n \in Z}$ implies $P_{2} x_{0}=0$ (respectively $P_{1} x_{0}=0$ ), that is, $x_{0}=0$.

If $P_{1} x_{0} \neq 0$ and $P_{2} x_{0} \neq 0$ then $\sup _{n>0}\left\|A_{1}^{n} P_{1} x_{0}\right\|<\infty$ while $\left(A_{2}^{n} P_{2} x_{0}\right)_{n \geqslant 0}$ is unbounded and hence, $\left(x_{n}=A_{1}^{n} P_{1} x_{0}+A_{2}^{n} P_{2} x_{0}\right)_{n \in Z}$ is unbounded.

Proposition 2.2. Let $A$ be a bounded linear operator from $X$ into itself, with the property $0 \notin \sigma(A)$. The linear discrete time system $(A, f)_{d}$ has for each

$$
f=\left(f_{n}\right)_{n \in Z} \in \ell^{\infty}(\mathbb{Z}, X)
$$

a unique solution

$$
x=\left(x_{n}\right)_{n \in Z} \in \ell^{\infty}(\mathbb{Z}, X)
$$

if and only if $\sigma(A) \cap C_{1}=\emptyset$.

ProOF: Let us suppose that for every $f \in \ell^{\infty}(\mathbb{Z}, X)$ the system $(A, f)_{d}$ has a unique bounded solution $\left(x_{n}\right)_{n \in Z}$. In particular, for every fixed $y \in X$ and for each $a \in C_{1}$ the system

$$
x_{n+1}=A x_{n}-a^{n-1} y
$$

has a unique bounded solution.

By the substitution $x_{n}=a^{n-1} z_{n}$ the system (2.1) becames

$$
z_{n+1}=a^{-1} A z_{n}-a^{-1} y
$$

It follows from here that $\left(x_{n}\right)_{n \in Z}$ is a solution for (2.1) if and only if

$$
\left(z_{n}\right)_{n \in Z}=\left(a^{1-n} x_{n}\right)_{n \in Z}
$$

is a solution of (2.2); moreover $\left(x_{n}\right)_{n \in Z}$ is bounded if and only if $\left(z_{n}\right)_{n \in Z}$ is bounded. Since the system (2.1) has, for every $y \in X$ and $a \in C_{1}$, a unique bounded solution, it 
follows that the system (2.2) has a unique bounded solution too, for every $y \in X$ and $a \in C_{1}$.

For $a \in C_{1}$ let us denote by

$$
z_{y}=\left(z_{n}\right)_{n \in Z} \in \ell^{\infty}(\mathbb{Z}, X)
$$

the unique bounded solution of the discrete time system (2.2).

Now we remark that

$$
z_{n+k+1}=a^{-1} A z_{n+k}-a^{-1} y
$$

and therefore, for every $k \in \mathbb{Z},\left(z_{n+k}\right)_{n \in Z}$ is also a bounded solution of (2.2). If we use the uniqueness property, it follows that $z_{n+k}=z_{n}$, for every $n \in \mathbb{Z}$, and $k \in \mathbb{Z}$; In particular we obtain $z_{k}=z_{0}$, for all $k \in \mathbb{Z}$. Hence we deduce that the solution $z_{y}=\left(z_{n}\right)_{n \in Z}$ is given by a constant sequence, that is $z_{n}=z_{0}$ for every $n \in \mathbb{Z}$. By virtue of (2.2) we can write

$$
z_{0}=a^{-1} A z_{0}+a^{-1} y
$$

or equivalently

$$
(a I-A) z_{0}=y
$$

and hence, for every $y \in X$ we can find a unique element $z_{0}$ (which depend on $y$ ) such that $(a I-A) z_{0}=y$.

From the previous arguments that $a \in \rho(A)$ and taking into acount that $a \in C_{1}$ was arbitrary, we obtain $\sigma(A) \cap C_{1}=\emptyset$.

Conversely, let us now suppose that $\sigma(A) \cap C_{1}=\emptyset$. By virtue of Proposition 2.1 we know that the equation $(A)_{d}$ has no bounded solution on $\mathbb{Z}$, except the case $x_{0}=0$. If for a sequence $\left(f_{n}\right)_{n \in Z} \in \ell^{\infty}(\mathbb{Z}, X)$, the equation

$$
(A, f)_{d} \quad x_{n+1}=A x_{n}+f_{n}
$$

has two bounded solutions

$$
\left(x_{n}^{\prime}\right)_{n \in Z} \text { and }\left(x_{n}^{\prime \prime}\right)_{n \in Z}
$$

then $\left(x_{n}^{\prime}-x_{n}^{\prime \prime}\right)_{n \in Z}$ is a bounded solution of equation $(A)_{d}$ and hence $x_{n}^{\prime}=x_{n}^{\prime \prime}$ for every $n \in \mathbb{Z}$.

It remain to show that for each bounded sequence $f=\left(f_{n}\right)_{n \in \mathbb{Z}}$, the equation $(A, f)_{d}$ has a bounded solution.

Let us take $f=\left(f_{n}\right)_{n \in Z} \in \ell^{\infty}(\mathbb{Z}, X)$. By writing

$$
\sigma_{1}=\sigma(A) \cap D_{1}, \quad \sigma_{2}=\sigma(A) \cap \operatorname{Ext}\left(D_{1}\right)
$$


we have that $\sigma_{1} \cup \sigma_{2}=\sigma(A)$ and $\sigma_{1} \cap \sigma_{2}=\emptyset$. Therefore we can associate the spectral projections $P_{1}, P_{2}$ and the linear operators $A_{1}, A_{2}$ (as in Proposition 2.1); it is possible that $\sigma_{1}=\emptyset$ (or $\sigma_{2}=\emptyset$ ) and in this case $P_{1}=0$ (or $P_{2}=0$ ).

The function $G: \mathbb{Z} \rightarrow \mathcal{B}(X)$ defined by

$$
G(n)= \begin{cases}A_{1}^{n} P_{1} & \text { if } n \geqslant 0 \\ -A_{2}^{n} P_{2} & \text { if } n<0\end{cases}
$$

will be called the discrete Greens function associated with the operator $A$.

Now for each

$$
f=\left(f_{n}\right)_{n \in \mathbb{Z}} \in \ell^{\infty}(\mathbb{Z})
$$

we define:

$$
\begin{aligned}
x_{n}= & \sum_{j=-\infty}^{\infty} G(n-j-1) f_{j}=\sum_{j=-\infty}^{n-1} G(n-j-1) f_{j} \\
& -\sum_{j=n}^{\infty} G(n-j-1) f_{j}=\sum_{j=-\infty}^{n-1} A_{1}^{n-j-1} P_{1} f_{j}-\sum_{j=n}^{\infty} A_{2}^{n-j-1} P_{2} f_{j}
\end{aligned}
$$

and so we obtain a sequence $\left(x_{n}\right)_{n \in Z}$.

Since

$$
\begin{aligned}
A x_{n}+f_{n} & =A\left(P_{1}+P_{2}\right) x_{n}+\left(P_{1}+P_{2}\right) f_{n} \\
& =\left(A_{1} P_{1}+A_{2} P_{2}\right)\left(\sum_{j=-\infty}^{n-1} A_{1}^{n-j-1} P_{1} f_{j}-\sum_{j=-\infty}^{n-1} A_{2}^{n-j-1} P_{2} f_{j}\right) \\
& +\left(P_{1}+P_{2}\right) f_{n}=P_{1} f_{n}+\sum_{j=n}^{\infty} A_{1}^{n-j} P_{1} f_{j}-\sum_{j=n}^{\infty} A_{2}^{n-j} P_{2} f_{j}+P_{2} f_{n} \\
& =\sum_{j=-\infty}^{n} A_{1}^{n-j} P_{1} f_{j}-\sum_{j=n+1}^{\infty} A_{2}^{n-j} P_{2} f_{j}=x_{n+1}
\end{aligned}
$$

we conclude that $\left(x_{n}\right)_{n \in Z}$ is a solution of the equation $(A, f)_{d}$.

On the other hand

$$
\begin{aligned}
\left\|x_{0}\right\| & \leqslant\left\|\sum_{j=-\infty}^{n-1} A_{1}^{n-j-1} P_{1} f_{j}\right\|+\left\|\sum_{j=n}^{\infty} A_{2}^{n-j-1} P_{2} f_{j}\right\| \\
& \leqslant \sum_{j=0}^{\infty}\left\|A_{1}^{j} P_{1} f_{n-j-1}\right\|+\sum_{j=1}^{\infty}\left\|\left(A_{2}^{-1}\right)^{j} P_{2} f_{n-j-1}\right\| \\
& \leqslant\left(\left\|A_{1}^{j}\right\| \cdot\left\|P_{1}\right\|+\sum_{j=1}^{\infty}\left\|\left(A_{2}^{-1}\right)^{j}\right\| \cdot\left\|P_{2}\right\|\right) \cdot\|f\|_{\infty} \cdot
\end{aligned}
$$


Since

$$
\sigma\left(A_{1}\right) \subset \operatorname{int} D_{1}
$$

and

$$
\sigma\left(A_{2}^{-1}\right) \subset\left\{\frac{1}{\lambda}:|\lambda|>1\right\} \subset \text { int } D_{1}
$$

we can find two numbers $N_{1}, N_{2}>0$ and $a \in(0,1)$ such that

$$
\left\|A_{1}^{j}\right\| \leqslant N_{1} \cdot a^{j}, \quad\left\|\left(A_{2}^{-1}\right)^{j}\right\| \leqslant N_{2} \cdot a^{j} .
$$

Hence

$$
\left\|x_{n}\right\| \leqslant \max \left(N_{1}, N_{2}\right) \cdot(1+a) \cdot(1-a)^{-1} \cdot\|f\|_{\infty}
$$

for every $n \in \mathbb{Z}$. Hence $\left(x_{n}\right)_{n \in \mathbb{Z}}$ is a bounded solution of the equation $(A, f)_{d}$.

\section{BOUNDED SOLUTIONS OF LINEAR SYSTEMS WITH UNBOUNDED OPERATORS}

Throughout this section, $A$ will be a closed linear operator which generates a $C_{0}$-group $\{T(t)\}_{t \in \mathbb{R}}$ of bounded linear operators on a Banach space $X$.

PROPISITION 3.1. If for every function $f$, continuous and bounded on $\mathbb{R}$, the system (1.1) has a unique mild solution

$$
x(t)=T(t) x(0)+\int_{0}^{t} T(t-s) f(s) d s
$$

bounded on $\mathbb{R}$, then

$$
\sigma(A) \cap\{\lambda \in \mathbb{C}: \operatorname{Re}(\lambda)=0\}=\sigma(A) \cap i \mathbb{R}=\emptyset .
$$

Proof: For every $\beta \in \mathbb{R}$ and $y \in X$ we can consider the equations:

$$
\begin{aligned}
\left(A ;-e^{i \beta t} y\right) & \dot{x}=A x-e^{i \beta t} y \\
(A-i \beta I ;-y) & \dot{z}=(A-i \beta I) z-y .
\end{aligned}
$$

It is well known that $A-i \beta I$ generates the following $C_{0}$-group of bounded linear operators

$$
\{S(t)\}_{t \in \mathbb{R}}=\left\{e^{-i \beta t} \cdot T(t)\right\}_{t \in \mathbb{R}}
$$

and therefore, every solution $x(\cdot)$ of equation $\left(A ;-e^{i \beta t} y\right)$ is defined on $\mathbb{R}$ by

$$
x(t)=T(t) x(0)-\int_{0}^{t} T(t-s) e^{i \beta s} y d s
$$


Also, every solution of equation $(A-i \beta I,-y)$ is defined on $\mathbb{R}$ by

$$
z(t)=S(t) z(0)-\int_{0}^{t} S(t-s) y d s
$$

or

$$
z(t)=T(t) e^{-i \beta t} z(0)-\int_{0}^{t} T(t-s) e^{-i \beta(t-s)} y d s
$$

In particular, if $x(0)=z(0)=x^{0} \in X$, then

$$
e^{i \beta t} z(t)=T(t) x(0)-\int_{0}^{t} T(t-s) e^{i \beta s} y d s
$$

and therefore we have a one to one correspondence between the solutions of equations $\left(A ;-e^{i \beta t} y\right)$ and $(A-i \beta I:-y)$, given by the equality:

$$
x(t)=e^{i \beta t} z(t),
$$

for every $t \in \mathbb{R}$.

Moreover, from the previous equality it follows that this correspondence preserves the bounded solutions and therefore, by virtue of the hypothesis, for every $\beta \in \mathbb{R}$ and $y \in X$, the equation $(A-i \beta I ;-y)$ has a unique solution on $\mathbb{R}$.

Now, we fix the number $\beta \in \mathbb{R}$ and denote by $z_{y}(\cdot)$ the unique bounded solution on $\mathbb{R}$ of equation $(A-i \beta I ;-y)$. If $z_{y}(0)=x^{0} \in X$, then for every $\tau \in \mathbb{R}$, we have

$$
\begin{aligned}
z_{y}(t+\tau) & =S(t+\tau) x^{0}-\int_{0}^{t+\tau} S(t+\tau-s) y d s \\
& =S(t) S(\tau) x^{0}-\int_{-\tau}^{t} S(t-u) y d u \\
& =S(t)\left[S(\tau) x^{0}-\int_{-\tau}^{0} S(-u) y d u\right]-\int_{0}^{t} S(t-u) y d u
\end{aligned}
$$

It follows from here that the function

$$
t \rightarrow z_{y}(t+\tau)
$$

is a bounded solution on $\mathbb{R}$ for the equation $(A-i \beta I ;-y)$ and thus, by hypothesis,

$$
z_{y}(t+\tau)=z_{y}(t), \quad \forall t, \tau \in \mathbb{R}
$$


and hence

$$
z_{y}(t)=z_{y}(0)=x^{0}, \quad \forall t \in \mathbb{R} .
$$

Now we obtain that

$$
x^{0}=S(t) x^{0}-\int_{0}^{t} S(t-s) y d s, \quad \forall t \in \mathbb{R}
$$

or equivalently

$$
\frac{1}{t}\left[S(t) x^{0}-x^{0}\right]+\frac{1}{t} \int_{0}^{t} S(t-s) y d s \quad \forall t \neq 0
$$

Hence

$$
\lim _{t \rightarrow 0} \frac{1}{t} S(t) x^{0}-x^{0}=y
$$

so that

$$
x^{0} \in \mathcal{D}(A-i \beta I)=\mathcal{D}(A)
$$

and

$$
(A-i \beta I) x^{0}=y .
$$

From the above arguments it follows that for every $y \in X$, we can find a unique element $x^{0} \in \mathcal{D}(A)$ with the property $(A-i \beta I) x^{0}=y$ and hence $A-i \beta I: \mathcal{D}(A) \rightarrow X$ is a bijective map. This $(A-i \beta I)^{-1}$, which is closed and defined on the whole of $X$, belongs to the space $\mathcal{B}(X)$, that is, $i \beta \notin \sigma(A)$.

\section{REFERENCES}

[1] J.L. Dalecki and M. G. Krein, Stability of solutions of differential equations in Banach spaces, translations of Mathematical Monographs, 43 (American Mathematical Society, Providence, R.I., 1974).

[2] M.G. Krein, Lekcii po ustoigivosti rexenii differencialnyh uravnenii v Banahovom pronstranstve (Kiev, Inst. Mat. DAN SSSR, 1964).

[3] J.L. Massera and J.J. Schäffer, 'Linear differential equations and functional Analysis, I', Ann. of Math. 67 (1958), 517-573.

[4] O. Perron, 'Die stabilitätsfrege bei differentialgleichungen', Math. Z. 32 (1930), 703-728.

[5] E. Topuzu, 'On Z-transform and linear discrete time systems', Lucr. Sem. Mat. Fiz. al I. P. T. (1985), 47-48.

[6] K. Yosida, Functional analysis (Springer-Verlag, Berlin, Heidelberg, New York, 1965). 